\title{
Future GLAST observations of SNRs and PWNe
}

\author{
S. Funk* and the GLAST-LAT collaboration ${ }^{\dagger}$ \\ *2575 Sand Hill Road, Menlo Park, CA-94025, USA \\ ${ }^{\dagger}$ http://www-glast.stanford.edu/
}

\begin{abstract}
Shell-type Supernova remnants (SNRs) have long been known to harbour a population of ultra-relativistic particles, accelerated in the Supernova shock wave by the mechanism of Diffusive shock acceleration. Pulsar Wind Nebulae (PWNe) diffuse structures surrounding young pulsars - are also known to be a site of particle acceleration in the Galaxy. $\gamma$-rays above $100 \mathrm{MeV}$ provide a direct access to acceleration processes through the following channels: ultra-relativistic electrons emit $\gamma$ radiation through Inverse Compton scattering in ubiquitous photon fields (such as CMBR), protons emit $\gamma$-radiation through the decay of $\pi^{0}$ s, generated in hadronic interactions with Interstellar material such as gas clouds. The upcoming GLAST Large Area Telescope (LAT) will be operating in the energy range between $30 \mathrm{MeV}$ and $300 \mathrm{GeV}$ and will provide excellent sensitivity, angular and energy resolution in a so-far rather poorly investigated energy band to address important questions in our understanding of both shell-type SNRs and PWNe.
\end{abstract}

Keywords: Supernova remnant, Pulsar Wind Nebula, GLAST

PACS: $98.38 . \mathrm{Mz}, 98.58 . \mathrm{Mj}$, 98.70.Rz, 98.70.Sa, 95.55.Ka

\section{GLAST studies of shell-type Supernova Remnants}

SNRs have long been thought to accelerate charged particles to ultra-relativistic energies. In spite of recent detailed studies in X-rays and VHE $\gamma$-rays, the nature of the parent population responsible for the VHE $\gamma$-ray emission above $100 \mathrm{GeV}$ in SNRs remains elusive. In the $100 \mathrm{MeV}$ to $10 \mathrm{GeV}$ band EGRET did find a statistical association of $\gamma$-ray emission with shell-type SNRs, however, no individual shell-type SNR was unambiguously identified. GLAST has both the spectral and angular resolution to make the first detailed study of these object between $30 \mathrm{MeV}$ and $300 \mathrm{GeV}$.

Spectral Studies: The GLAST-LAT will provide measurements of $\gamma$-ray spectra between $30 \mathrm{MeV}$ and $300 \mathrm{GeV}$, a previously rather poorly-explored energy regime. Using LAT data will allow us to distinguish between different models for the $\gamma$-ray emission. $\gamma$-rays of leptonic origin (produced by IC) can be distinguished from those of hadronic origin through their characteristic spectral shape. H.E.S.S. measurements in VHE $\gamma$-rays above $100 \mathrm{GeV}$ already provide some constraints on the emission level for these different models. Fig. 1 (left) shows prediction for energy spectra for a hadronic and a leptonic model illustrating that the LAT energy range is particularly well suited to distinguish these models and possibly provide the first direct evidence of hadronic acceleration in the shells of SNRs.

Morphological Studies: The unprecedented GLAST-LAT angular resolution in this energy range will alleviate the problem of source confusion in the Galactic plane and will allow for studies of the $\gamma$-ray emission regions in the larger of the known $\gamma$-ray emitting SNRs, such as RXJ1713.7-3946 (Fig. 1 (right)). The angular resolution of the instruments improves with energy and thus young nearby SNRs with angular size $>1^{\circ}$ can be significantly resolved above $\sim 10 \mathrm{GeV}$. Correlation studies with hard X-rays, as well as with VHE $\gamma$-rays will give detailed views into the acceleration sites, providing energetic coverage over many orders of magnitude. The excellent angular resolution will isolate the shell-emission from the core PWN in composite SNRs and allow for population studies of shell-type SNRs in the $\gamma$-ray regime.

\section{GLAST studies of Pulsar Wind Nebulae}

EGRET found a number of bright variable Galactic objects possibly associated with PWNe. Recent advances in VHE $\gamma$-rays above $100 \mathrm{GeV}$ by H.E.S.S. have shown at least 8 PWNe emitting at $\gamma$-ray energies detected in a survey of the southern Galactic plane. Many of these are expected to be detectable with GLAST. The SED for bright pulsars should be dominated in the $\mathrm{GeV}$ range by the central pulsar (as e.g. in the Crab). However, phase analysis can cut 

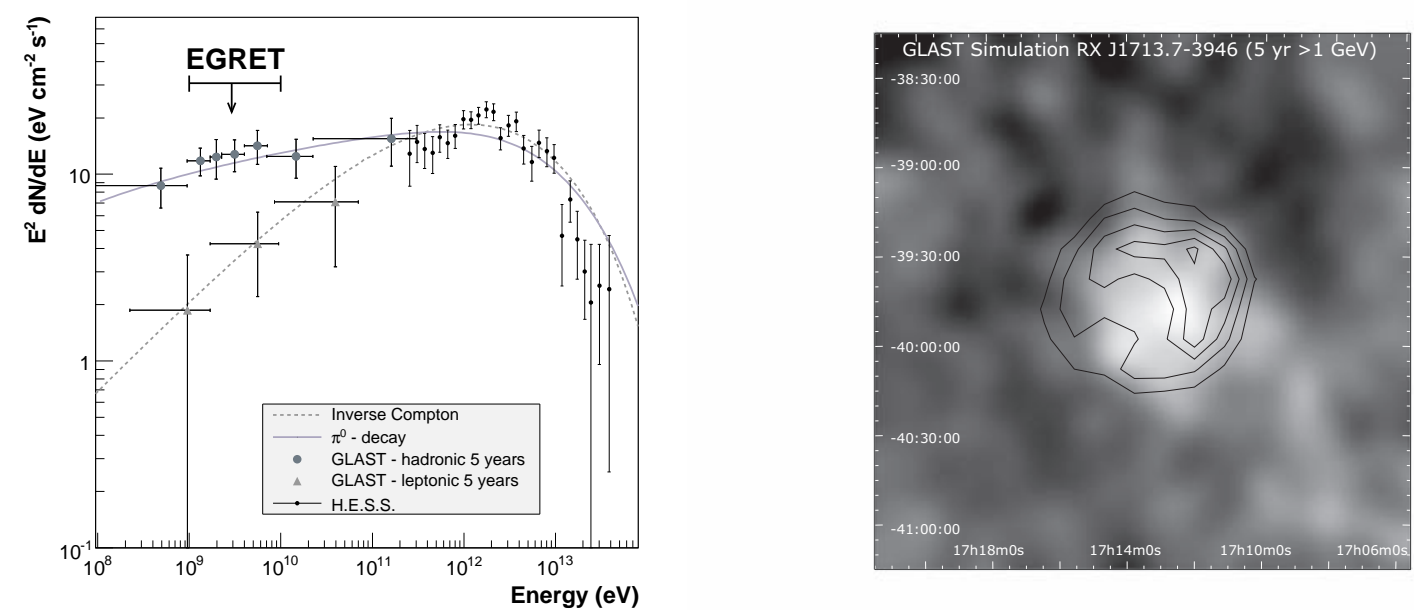

FIGURE 1. Left: Spectral energy distribution of the shell-type SNR RX J1713.7-3946 above $100 \mathrm{MeV}$. The black data points denote measurements with H.E.S.S., the dark grey and light grey data points correspond to simulated GLAST data assuming different mechanisms for the $\gamma$-ray emission. Please note that this simulation uses the current best estimate of the LAT performance.Right: Simulated map for RX J1713.7-3946 in a hadronic model above $1 \mathrm{GeV}$ as seen by GLAST in 5 years of observation along with the H.E.S.S. contours in white. A very simple smoothing algorithm has been applied and a more detailed analysis might resolve the shell-like emission in this object.

out the pulsed emission, revealing the $>100 \mathrm{MeV}$ PWN spectrum. GLAST will be able to determine morphologies and energy spectra for a large number of PWNe and allow for detailed population studies. The nearly continuous coverage and stable high sensitivity of GLAST should allow us to measure the slow (monthly) variability of the PWN synchrotron component from the wind termination shock providing a new probe of PWN dynamics.

\section{Best Candidates for GLAST detections}

Shell-type SNRs: With our current knowledge, the best candidates for finding $\gamma$-ray emission in both shell-type SNRs and PWNe with GLAST will have to obey the following criteria: a) detection in VHE $\gamma$-rays and b) hard $\mathrm{X}$-ray (synchrotron emission) pointing to non-thermal particle populations c) possible association with an EGRET unidentified source. The most promising candidates for GLAST detections are summarised in Tab. 1.

TABLE 1. SNR and PWN candidates for GLAST.

\begin{tabular}{cc}
\hline Shell-type Supernova Remnants & Pulsar Wind Nebulae \\
\hline RX J1713.7-3946 & Crab \\
RX J0852.0-4622 & Vela X \\
Cas A & MSH 15-52 \\
SN 1006 & Kookaburra \\
RCW 86 & PSR B1706-44 \\
Tycho SNR & PSR B1823-13 \\
Kepler SNR & MSH 11-54 \\
IC 443 & 3C 58 \\
W 28 & W44 \\
\hline
\end{tabular}

\section{Summary}

The prospects for GLAST of detailed investigations of SNRs and PWNe promise to provide a sensitive new probe of particle acceleration mechanisms in our Galaxy. Measurements in adjacent X-ray and VHE $\gamma$-ray energy bands allow for detailed predictions of possible $\gamma$-ray signatures in the GLAST energy range. Best candidates for a $\gamma$-ray detection with GLAST are thus young SNR and young to mid-aged PWNe already detected in VHE $\gamma$-rays or exhibiting hard $\mathrm{x}$-ray synchrotron emission. 